\title{
A Collaborative E-learning Component for the IDEFIX Project
}

\author{
T. Hernán Sagástegui Ch., José E. Labra G., Juan M. Cueva L., \\ José M. Morales G., María E. Alva O., Eduardo Valdés, and Cecilia García \\ University of Oviedo, Department of Computer Science, Calvo Sotelo S/N, \\ 33007 Oviedo, Spain \\ \{thsagas, jmmoral, malva360\}@correo.uniovi.es, \\ \{labra, cueva\}@lsi.uniovi.es \\ eduardovr@telecable.es, cgpelayo27@hotmail.com
}

\begin{abstract}
This paper presents the design and architecture of a collaborative learning model based on the Web as an experience that it is being done at the University of Oviedo, giving support to the students of a logic course, in the collaborative achievement of exercises by means of e-meetings and in the training of the achievement of exams by means of a virtual reality game. This model is developed like a component of the project IDEFIX and it uses Web services.
\end{abstract}

\section{Introduction}

The IDEFIX project (Integrated Development Environment Frameworks based on Internet and eXtensible technologies) is based on the development of e-learning tools based on the use XML and related technologies [1]. IDEFIX can extend its domain to the teaching for Internet of any course at the School of Computer Science Engineering at Oviedo (EUITIO), as well as to the shared teaching of free election courses through Internet in the AulaNet project [2] of other universities. In this context, this article shows an extension component of the IDEFIX project. This component is referred to the design and architecture of collaborative learning model of the logic course through the Web.

At this moment most of the students attend the course in the classroom and it has a didactic guide of the classes and a notebook that gathers the exams carried out in the convocations of previous years that are used as exercises as pedagogic material of support.

The goal is that the students can access the notebook of exercises by Internet and that they can solve these exercises in a collaborative way, and that they can also fill their exams by means of a virtual reality game through the web.

\section{Background}

Computer supported collaborative learning (CSCL) supports group work in a common task and provides a shared interface for groups to work with [3], [4],[12], by means of a net of computers, supporting the coordination, and the application of the 
knowledge in certain domains [5], [6]. CSCL is used in the educational environment and it serves from support to the students in the learning, facilitating the group working process and group dynamics in ways that are not achievable by face-to-face learning [12].

There are numerous commercial collaborative applications that support: problems resolutions, tutorials, simulations [9], debates, modeling and CourseWare (Blackboard: Virginia Commonwealth University, LearningSpace: Lotus \& IBM [11], WebCT: University British Columbia, TopClass: WBT Systems [10], etc), while for the learning of the logic there is projects like JAPE [7], [8]. Collaborative application inside an outline of group working has the following characteristic: group memory, roles, collaboration protocol, perception and interface [3], [5].

\section{Model of Collaborative Learning}

The model of collaborative learning for the logic course is based on the group and on the e-meetings of the group, in a distributed environment, being able to design a cycle of life of the e-meetings [3],[4],[5],[6]. The elementary components of the model are:

Group Memory: it is formed by the database of the university (with links to courses and professors), the base of exercises/exams and the games.

Roles: the professor, the student that develops exercises the student that solves the exam and the invited user.

Collaboration Protocols: rules settled down by the professor, rules for the achievement of exercises and exams, rules for the invited users and rules for the administrator.

Perception: it is the information generated in the achievement of assignments and exams; which members of the group are connected, which exercises are developed, obtained results, generation of ideas, taking of decisions, using the chat as main tool.

Interface: It allows the user to interact with the group memory and with the generated knowledge, and to select the work (Collaborative development of the exercises of the exercises notebook, and to carry out training exams).

\section{Architecture of the Model}

The architecture for the implementation of this model is based on a 3 layer client/server design presented in Figure 1. It is implemented as a component of the IDEFIX project on Microsoft .NET platform.

\subsection{Presentation Layer}

This layer presents the user interface and takes charge of the visualization of the clients and the data entry. Once the client makes its petitions from the navigator, the Web server takes the petition to an ASP page. 


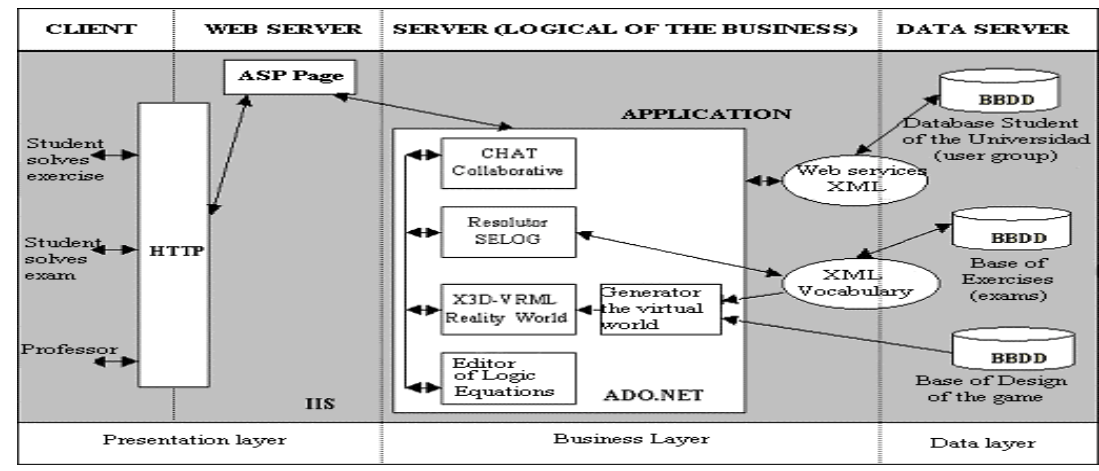

Fig. 1. This graph shows the architecture of design of the application web, corresponding to a structure client-server, where the application layer or business layer it shows the support that will give to the collaborative component for the process of collaborative learning.

\subsection{Business Layer}

This layer is in charge of the logic of the application and the access to the data. It is formed by:

Logic equations editor: Similar to that of Microsoft Word, it facilitates the creation of logic equations storing the results in an XML specific vocabulary.

Collaborative CHAT: query chat and feedback chat.

SELOG Solver: It allows the collaborative development of logic exercises. It presents an interface and carries out: selection of exercises, solution, consults and evaluation of the difficulty and comments. Chat is mainly used for group work.

Virtual Reality World: It is designed as support to the students in the training and achievement of exams. The micro-world of logic - Logical world - is a world formed by spheres that describe the different levels of the game. At this moment, to pass from one sphere to another, it is necessary to approve all the phases of the corresponding level.

Starting from an XML document and common data from the database a program written in $\mathrm{C} \#$ generates the virtual world in $\mathrm{X} 3 \mathrm{D}$, which can automatically be converted to VRML.

\subsection{Data Layer}

This layer stores data of the students, all the exercises/exams and the games and data that the generator of the virtual world needs. 


\section{Conclusions}

This work contributes to the development of collaborative e-learning applications for secondary and higher education [13] that satisfy the communicative requirements and pedagogic features of these educational levels. It also supports the development of elearning collaborative applications. Although the collaborative application is in development and the prototypes have an autonomous functionality; it is designed to become a components of the IDEFIX project in its scalable development. Future research will be managed to implement the e-learning collaborative application in other courses at the School of Computer Science Engineering of Oviedo.

\section{References}

1. Labra, J., Morales, J., Fernandez, A., Sagástegui, H.: A Generic e-Learning Multiparadigm Programming Language System: IDEFIX Project. SIGCSE'03 USA (2003)

2. Pérez, R., López, A.: Aulanet, una Experiencia de Aula Virtual Spain (2000)

3. Ellis, C., Gibbs, S., Rein, G.: Groupware some issues y experiences, Comm. of the ACM, Vol. 34 No. 1 (1991) 38-58

4. Conklin, J.: Capturing Organizational Memory. Readings in Groupware and ComputerSupported Cooperative Work. Morgan Kaufmann Publishers CA (1993) 561-565

5. Guerrero, L. Fuller, D.: CLASS: A Computer Platform for the Development of Education's Collaborative Applications. Proceeddings of CRIWG'97, $3^{\text {rd }}$ International Workshop on Groupware Spain (1997) 1-3

6. Gokhale, A.: Collaborative Learning Enhances Critical Thinking. Journal of Technology Education, Vol. 7, № 1, Fall 1995, University Libraries Virginia (1996)

7. Aczel, J.: The Evaluation of a Computer Program for Learning Logic: The Role of Students' Formal Reasoning Strategies in Visualising Proofs. CALRG Technical Report (2000) 192

8. The Jape Visualisation Project http://iet.open.ac.uk/pp/j.c.aczel/Jape/index.html

9. LEGO Mindstorms http://mindstorms.lego.com/eng/default.asp

10. TopClass e-Learning Suite $\mathrm{TM}^{\mathrm{TM}}$ http://www.wbtsystems.com/products

11. IBM Lotus Software http://www.lotus.com/home.nsf/tabs/learnspace

12. Lin, W.: CSCL Theories. Texas University. USA (1996) http://www.edb.utexas.edu/csclstudent/Dhsiao/theories.html

13. Computer-Supported Intentional Learning Environments http://www.ed.gov/pubs/EdReformStudies/EdTech/csile.html 